\title{
Unveiling the Core of M 15 in the Far-Ultraviolet
}

\author{
A. Dieball ${ }^{1}$, C. Knigge ${ }^{1}$, D. R. Zurek ${ }^{2}$, M. M. Shara ${ }^{2}$, K. S. Long ${ }^{3}$, \\ P. A. Charles ${ }^{4}$ and D. Hannikainen ${ }^{5}$ \\ ${ }^{1}$ Department of Physics and Astronomy, University Southampton, SO17 1BJ, UK \\ email: andrea@astro.soton.ac.uk \\ ${ }^{2}$ Department of Astrophysics, American Museum of Natural History, New York, NY 10024 \\ ${ }^{3}$ Space Telescope Science Institute, Baltimore, MD 21218 \\ ${ }^{4}$ South African Astronomical Observatory, PO Box 9, Observatory, 7935, South Africa \\ ${ }^{5}$ University of Helsinki, P.O. Box 14, SF-00014 Helsinki, Finland
}

\begin{abstract}
We present an analysis of our deep far- (FUV) and near-ultraviolet (NUV) photometry of the core region of the dense globular cluster M 15. Our FUV-NUV colour-magnitude diagram (CMD) is the deepest one presented for a globular cluster so far, and shows all hot stellar populations expected in a globular cluster, such as horizontal branch stars, blue stragglers, white dwarfs, cataclysmic variables and even main sequence stars. The main sequence turn-off is clearly visible and the main sequence stars form a prominent track that extends at least two magnitudes below the main sequence turn-off. We compare and discuss the radial distribution of the various stellar populations that show up in the FUV. We search for variability amongst our FUV sources and tentatively classify our variable candidates based on an analysis of the UV colours and variability properties. We find that RR Lyraes, Cepheids, and SX Phoenicis exhibit massive variability amplitudes in this waveband (several mags).
\end{abstract}

Keywords. globular clusters: individual (M 15), stars: cataclysmic variables, white dwarfs, stars: variables: other, ultraviolet: stars, blue stragglers, binaries: close

Stellar densities in the cores of globular clusters (GCs) are extremely high, so that dynamical encounters between the cluster stars are inevitable, leading to a variety of exotic stellar populations like blue stragglers (BSs), cataclysmic variables (CVs), lowmass X-ray binaries (LMXBs) and other close binary systems. These exotica all show a spectral energy distribution bluer than ordinary main sequence stars and red giants. As a consequence, crowding is considerably reduced, making FUV observations the ideal tool to study these dynamically-formed stellar populations in the dense cluster cores.

\section{Results:}

Our study confirms that FUV observations are particularly well suited in studying hot and especially dynamically formed stellar populations. We detected prominent populations of $\mathrm{CV}, \mathrm{BS}$ and WD candidates and $\mathrm{HB}$ stars. CV and BS candidates are the most centrally concentrated stellar populations, which might either be an effect of mass segregation or reflect the preferred birthplace in the dense cluster core of such dynamicallyformed objects. We found in total 41 variable sources. RR Lyrae, Cepheids and SX Phoenicis stars are easily detected as they show large FUV amplitudes. This is expected since the pulsation amplitude increases towards the FUV, but this is the first time they are actually observed for SX Phoenicis stars. 

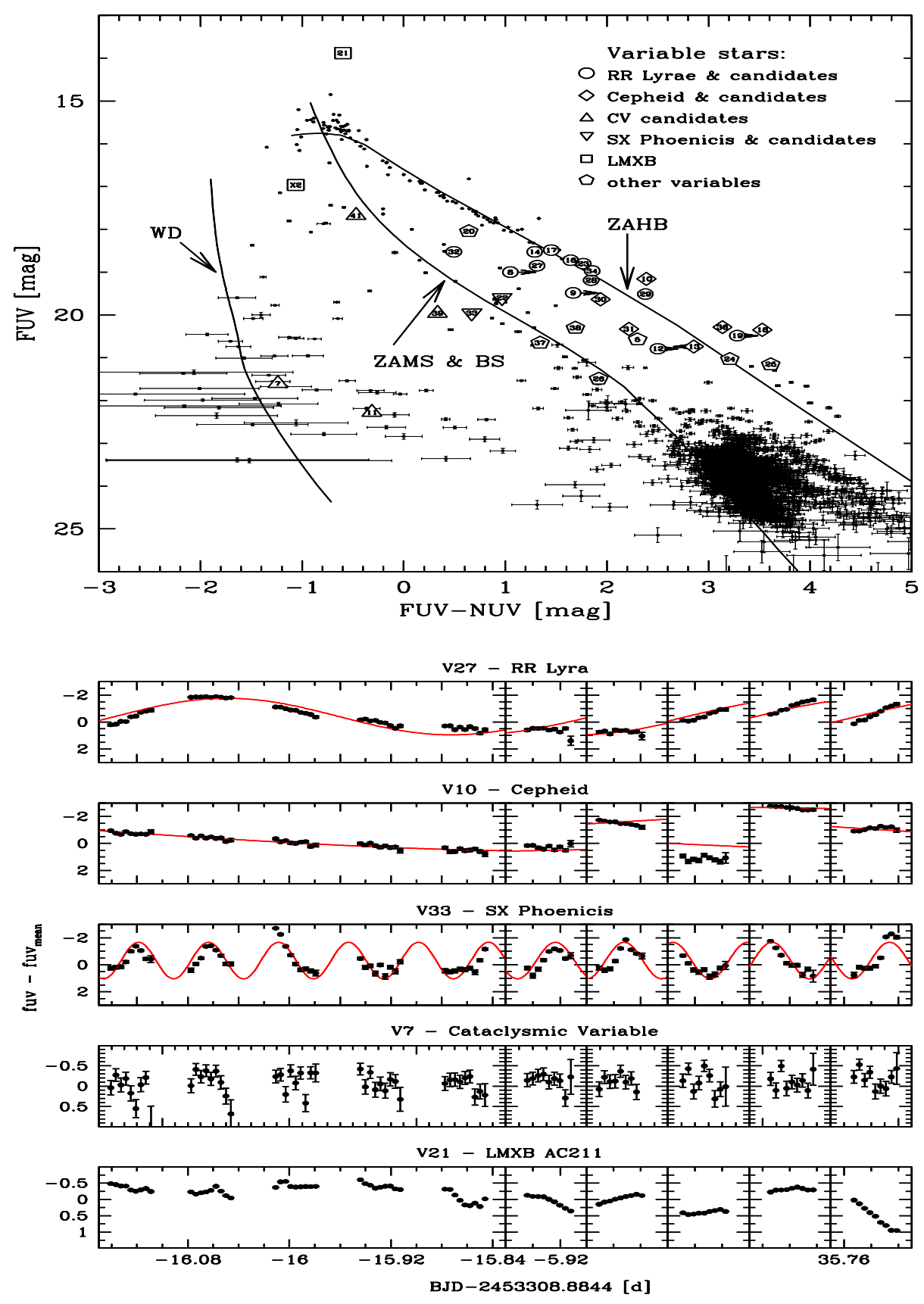

Figure 1. Top: Several stellar populations show up in our FUV-NUV CMD, such as the bright HB stars, BS and WD candidates. CVs and detached WD-MS binaries are located between the WD cooling sequence and the MS. The clump of MS stars and red giants reaches $\approx 2$ mag below the cluster's turn-off. As such, this is the deepest FUV-NUV CMD presented for a GC so far. Bottom: We found in total 41 variable sources and suggest a classification based on their light curves and location in the CMD. We find four previously known RR Lyrae and 13 further candidates, one known Cepheid and six further candidates, one known and one probable SX Phoenicis star, six CVs, and the well known LMXB AC 211. 